\title{
A practical appraisal method for through-wall cracks in tubular structures
}

\author{
S. Limkatanyu ${ }^{1} \&$ K. Kuntiyawichai ${ }^{2}$ \\ ${ }^{I}$ Department of Civil Engineering, Prince of Songkla University, \\ Songkhla, Thailand \\ ${ }^{2}$ Department of Civil Engineering, Ubonratchathani University, \\ Ubonratchathani, Thailand
}

\begin{abstract}
Tubular structures such as offshore platforms are used for various reasons, i.e. oil and gas exploration, navigation aid towers, bridges and causeways, ship loading and unloading facilities. These structures are mostly made of various grades of steel, from mild to high strength, i.e. $240 \mathrm{MPa}$ to $360 \mathrm{MPa}$ yield. In order to design members or components of tubular structures under static loads to avoid failure, collapse and buckling etc., design codes and standards are well established for specific applications, e.g. BS5950, BS5400 and EuroCode 3. However, the conventional design criteria are insufficient for designing engineering structures not containing flaws, since all structures contain flaws that may be caused by the manufacturing processes, fabrication or due to localized in-service damage. These flaws may grow by fatigue due to the application of repeated loads and this could lead to degradation in the structural strength with the possibility of catastrophic failures. Therefore, in order to perform a fracture assessment of tubular connections containing through-wall cracks of tubular structures, a 3D-submodel analysis of the selected connection has to be analyzed using full-scale dynamic finite element analyses, which is time is consuming. Hence, high performance computing facilities are required which are beyond the scope of conventional designers. Thus, a simplified method for estimating the performance of the tubular connection is introduced in this study. The results from the approximate method are then compared with those obtained from the full dynamic FE analyses.

Keywords: tubular structures, failure assessment, crack connections, finite element analysis.
\end{abstract}




\section{Introduction}

In general, there are two basic types of offshore oil/gas exploration (and drilling) platforms, i.e. moveable platforms and permanent platforms. Moveable rigs are often used for exploratory purposes because they are much cheaper to use than permanent platforms. Once large deposits of hydrocarbons have been found, a permanent platform is built to allow their extraction. These structures are mostly made of various grades of steel, from mild to high strength, i.e. $240 \mathrm{MPa}$ to 360 $\mathrm{MPa}$ yield. In order to design members or components of tubular structures under static loads to avoid failure, collapse and buckling etc., design codes and standards are well defined including BS5950 [1], BS5400 [2], EuroCode 3 [3] and equivalent codes in other countries, whilst for offshore structures the design code used almost is invariably API [4].

However, the conventional design criteria are insufficient to design engineering structures not containing flaws since all structures contain flaws that may be caused by the manufacturing processes, fabrication or due to localized in-service damage. These flaws may grow by fatigue due to the application of repeated loads and this could lead to degradation in the structural strength with the possibility of catastrophic failures. Moreover, there is little or no guidance on fracture assessment for dynamic loaded structures, members, or components. Some codes warn that the frequency of dynamic loading should avoid coincidence with structural natural frequencies but without giving any detailed guidance. This leads to the conventional assessment method for assessing crack tip severity of cracked structures, which has been studied by the authors [5-7] (for detailed explanations please see the next section). However, the conventional assessment method is time consuming and high performance computing facilities are required which are beyond the scope of conventional designers.

Therefore, this paper attempts to represent the real situation by simplified treatment to provide guidance on practical assessment methods. A simplified method for estimating the performance of the tubular connection is introduced in this study. The results from the approximate method are then compared with those obtained from the full dynamic FE analyses.

\section{Conventional method for assessing through-wall cracks in tubular structures}

In order to perform an assessment of the crack tip severity in engineering structures using conventional methods, Kuntiyawichai and Burdekin [5] introduced the engineering assessment of crack structures, especially beam-column connections subjected to earthquake loading using fracture mechanics assessment. The results showed good agreement with the crack obtained at the damaged buildings during the Northridge earthquake. After that a similar approach was performed on minimum structures that had a different connection shape, crack shape and applied loading on structures [6, 7]. The main procedures of both studies can be summarized below. 
Step 1: Dynamic analysis of structures: In general, dynamic analysis involves the determination of the response of a structure or component that is subjected to forces or displacements that vary with time. Hence, by performing dynamic analyses, the dynamic characteristic of tubular structures, especially the frequency response, magnitude and location of maximum stress (or the worst location) and potential resonance of tubular structures, can be obtained. Figure 1 shows an example of a FE model for performing dynamic analysis of structures.

Step 2: Submodel analysis of structures: In this step, the local behaviour of connections with the assumed defect within the complete FE model of tubular structures is investigated. For the 3D submodel connection, a three-dimensional shell or solid elements incorporating initial cracks are modeled. Although the submodel with a 3D shell or solid element within the full 3D beam element model proved to be too computationally expensive, it provided a valuable understanding of the local behaviour of connections situated in the complete brace caisson. Time consuming and high performance computing facilities are required in this step.

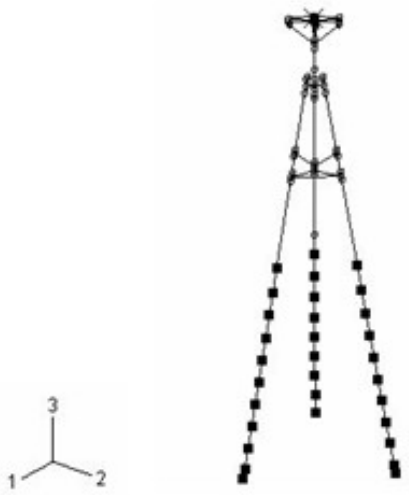

Figure 1: An example of a FE model for dynamic analysis of structures.

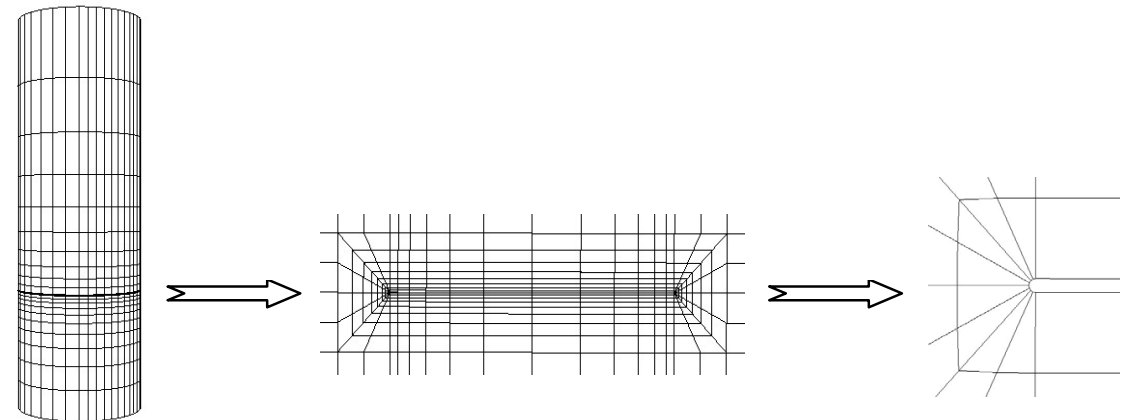

Figure 2: $\quad$ Example of a 3D submodel of a cracked connection. 
Step 3: Extracting the crack tip characterizing parameter: After performing submodel analysis, the crack tip characterizing parameter, i.e. CTOD $\left(\delta_{I}\right)$ is obtained. This parameter can be used to evaluate the crack tip severity of the connection. There are two common ways to define the crack tip opening displacement (CTOD), $\delta_{I}$, namely the displacement at the original crack tip and the $90^{\circ}$ intercept, as shown in Figure 3.

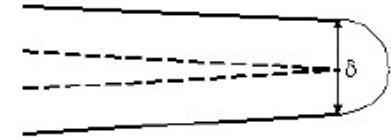

(a) Displacement at the original crack tip

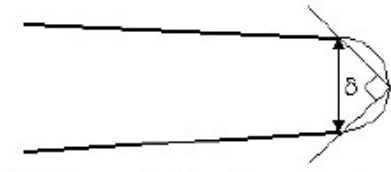

(b) Displacement at the intersection of a $90^{\circ}$ vertex with the crack lentss

Figure 3: Definitions of CTOD [8].

Based on the procedures described above, all procedures, especially step 2, time consuming and high performance computing facilities are required which is beyond the scope of conventional designers. Thus, a practical assessment for estimating the performance of the tubular connection is introduced in this study.

\section{Practical assessment methods for through-wall cracks in tubular structures}

From the point of view of assessment of structural integrity of structures containing cracked, a close approximation to the practical side of the problem can be obtained from the fracture mechanics assessment. Based on the stresses occurring at the members, the method for assessing the crack tip severity at the crack tip can be characterized into two cases, i.e. stress-based concepts and strain-based concepts respectively. The details of each concept are shown in the following section.

\subsection{Stress-based concepts}

When the maximum stresses at the members are below yield strength or contained by elastic regions, the stress-based concepts can be used in the assessment of safety critical components. The BSI Document PD 6493 [9] has been most widely used by following that procedure and is a major part of the latest version of that code given in BS7910 [10]. The applied stress intensity factor, $K_{I}$ has the following general form:

$$
K_{I}=(Y \sigma) \sqrt{\pi a}
$$

where 


$$
Y \sigma=M f_{w} M_{m} \sigma_{\max }
$$

and where $a$ is half flaw length of through-thickness flaw; $M$ is bulging correction factor; $f_{w}$ is finite width correction factor; $\sigma_{\max }$ is the maximum tensile stress; $M_{m}$ is a stress intensity magnification factor.

The applied CTOD, $\delta_{I}$, is determined from $K_{I}$ as follows:

- For steels (including stainless steels) and aluminium alloys where $\sigma_{\max } / \sigma_{Y} \leq 0.5$, and for all $\sigma_{\max } / \sigma_{Y}$ ratios with other materials:

$$
\delta_{I}=\frac{K_{I}^{2}}{\sigma_{y} E}
$$

- For steels (including stainless steels) and aluminium alloys where $\sigma_{\max } / \sigma_{Y}>0.5$ :

$$
\delta_{I}=\frac{K_{I}^{2}}{\sigma_{y} E}\left(\frac{\sigma_{y}}{\sigma_{\max }}\right)^{2}\left(\frac{\sigma_{\max }}{\sigma_{y}}-0.25\right)
$$

where $\sigma_{Y}$ is yield strength of the material and $E$ is modulus of elasticity of the material.

\subsection{Strain-based concepts}

When the problem involves extensive deformation and high plastic strains, a strain-based method can be derived as follows [11]:

$$
J=2 F^{2} \pi \sigma_{u n c} \varepsilon_{u n c}
$$

where $\sigma_{u n c}$ is the stress in the uncracked structure, $\varepsilon_{u n c}$ is the strain in the uncracked structure, $a$ is the crack depth, and $F$ is the geometric magnification factor for evaluating stress intensity factor.

Referring to the relationship between $\mathrm{J}$ and CTOD Equation (5) can be written as:

$$
\operatorname{CTOD}\left(\delta_{I}\right)=\frac{F^{2} \pi a \sigma_{u n c} \varepsilon_{u n c}}{\sigma_{Y}}
$$




\section{Numerical examples}

One type of minimum structures namely braced caisson (Figure 1) was modeled in this study. The platform was designed by Ramboll [12] according to API [4]. The brace caisson has the total height of 52 metres measured from the seabed and a pile penetration of 48 metres. The top deck of the braced caisson has a 400 metric tons mass. A three-dimensional, three-noded quadratic beam element, i.e. B32, (as recommended in Ultiguide [13]) was used to model the platform tubular members. These elements have 16 integration points around the circumference. Each element was rigidly attached to each other. Material properties used for the FE model are shown below:

$\begin{array}{ll}\text { Young's modulus } & 205 \times 10^{9} \mathrm{~N} / \mathrm{m}^{2} \\ \text { Yield stress } & 396.75 \times 10^{6} \mathrm{~N} / \mathrm{m}^{2} \\ \text { Density of steel } & 8242.75 \mathrm{~kg} / \mathrm{m}^{3}\end{array}$

The elements used for the modeling of sub models were the general-purpose three dimensional shell elements, i.e. S4R available in ABAQUS [14]. These elements have six degrees of freedom per node. With an adequately fine mesh, these elements are capable of providing accurate solutions even in complex structures. For the crack tip region, an absolute sharp crack tip should not be adopted in large strain analysis because it may cause stress singularity. Therefore a crack tip with initial root radius was introduced in this modeling. The initial root radius was assumed to be $0.03 \mathrm{~mm}$ in all cases of study in order to prevent the overlap between the crack faces. The dimensions of the tubular joint are $2.134 \mathrm{~m}$ diameter and $30 \mathrm{~cm}$ and $31 \mathrm{~cm}$ for the thickness of the top part and the bottom part of the connection respectively. In order to study the effect of initial crack length on applied CTOD and stress distribution pattern, five initial crack lengths, i.e. no crack, $2.5 \mathrm{~cm}, 5 \mathrm{~cm}, 10 \mathrm{~cm}$ and $33 \mathrm{~cm}$, were assumed to locate in the global X direction (the direction of applied wave loading).

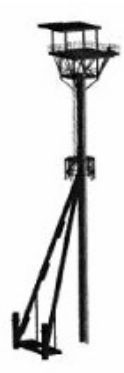

(a)

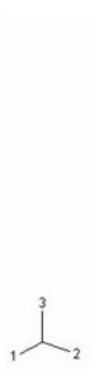

(b)
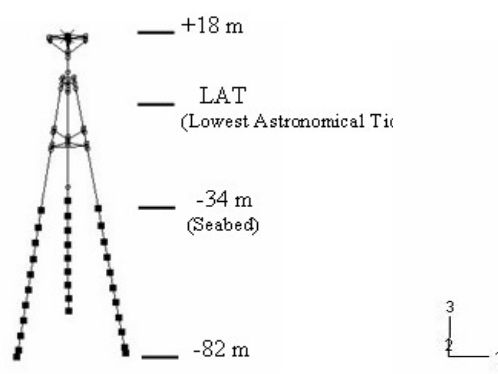

(c)

Figure 4: (a) Minimum structures (b) FE model (c) FE model with 3D submodel. 
By using the above equations, a simplified method for assessing the crack tip severity can be done by performing the following procedures:

- $\quad$ Perform dynamic time history analyses on a simple brace caisson model subjected to wave loading (model containing only beam element).

- Extract the maximum stress value from the critical connection.

- Substitute all data in either Equation (3) or Equation (4) depending on $\sigma_{\max } / \sigma_{Y}$ ratio for the case of maximum stresses at the members below yield strength and Equation (6) when the problem involves extensive deformation and high plastic strains.

From a simplified FE analysis (Figure 4b), the stress level at the same connection, as in the submodel analyses (Figure 4c), was extracted from the original FE model, which was taken as $200 \mathrm{MPa}$. It could be seen that the ratio between $\sigma_{\max } / \sigma_{Y}$ is equal to 0.5. Hence, Equation (3) was used to perform the simplified analyses. By applying the simplified method, the applied CTOD for different crack lengths can be predicted as summarized in Table 1.

Table 1: $\quad$ Comparison of the applied CTOD values.

\begin{tabular}{|c|c|c|}
\hline \multirow{2}{*}{$\begin{array}{c}\text { Crack length } \\
(\mathrm{cm})\end{array}$} & \multicolumn{2}{|c|}{ CTOD (mm) } \\
\hline & $\begin{array}{c}\text { Submodel Analyses } \\
{[6]}\end{array}$ & Simplified Analyses \\
\hline 2.5 & 0.017 & 0.0193 \\
\hline 5 & 0.0225 & 0.0306 \\
\hline 10 & 0.041 & 0.0548 \\
\hline 33 & 0.125 & 0.145 \\
\hline
\end{tabular}

The results in Table 1 are considered as the critical approximation of applied CTOD. It can be seen that the results of applied CTOD give a good agreement with the FE results of submodel analyses. Hence, this method can enormously reduce the analysis time which allows design engineers to assess the possibility of connection fractures, or to determine approximate values of toughness and defect size requirements for given peak stress levels.

\section{Conclusions}

The practical assessment method for through-wall crack in tubular structures subjected to wave loading has been introduced. The applied CTOD values obtained from a simplified method showed a good agreement with the FE results of submodel analyses. Therefore, this method can enormously reduce the analysis time which allows design engineers to assess the possibility of connection fractures, or to determine approximate values of toughness and defect size requirements for given peak stress levels. 


\section{Acknowledgements}

This project was supported by Ministry of University Affairs (MUA) and Thailand Research Fund (TRF) under Grant Number MRG4680060. The support is gratefully acknowledged.

\section{References}

[1] BS5950:2000, Design of Steel Buildings, British Standard Institution, London, 2000.

[2] BS5400:2000, Design of Steel and Composite Bridges, British Standard Institution, London, 2000.

[3] EuroCode 3., European Code for Design of Steel Buildings.

[4] API., API RP 2A Recommended practice for planning, designing and constructing fixed offshore platforms, $20^{\text {th }}$ edition, and supplement 1 , American Petroleum Institute, Washington, DC, USA, 1996.

[5] Kuntiyawichai, K. \& Burdekin, F.M., Engineering assessment of cracked structures subjected to dynamic loads using fracture mechanics assessment. Engineering Fracture Mechanics, 70, pp. 1991-2014, 2003.

[6] Kuntiyawichai, K. \& Chucheepsakul, S., Assessment of through-wall crack in Minimum structures subjected to wave loading. Engineering Structures, 2006, 28(11), pp. 1533-1542, 2006.

[7] Kuntiyawichai, K., Assessment of fracture in offshore structures subjected to wave loading, MUA-TRF final report MRG4680060, Thailand Research Fund, Bangkok, Thailand, 2005.

[8] Anderson, T.L., Fracture Mechanics: Fundamentals and Applications, CRC Press, London, 1995.

[9] PD6493:1991, Guidance on methods for assessing the acceptability of flows in fusion welded structures, British Standard Institution, London, 1991.

[10] BS7910:1999, Guide on methods for assessing the acceptability of flows in fusion welded structures, British Standard Institution, London, 2000.

[11] Linken, D., Formby, C.L. \& Ainsworth, R.A., Strain-based approach to fracture assessment-example applications. Proceedings of the fifth international conference on engineering structural integrity assessment, Cambridge, UK; 2000.

[12] Ramboll, JIP Comparative Evaluation of Minimum Structures and Jackets, Issue 6, Document Ref:AM7565/JIPAGR. Atkins Research \& Development, Surrey, UK, 1998.

[13] Ultiguide, Best Practice Guidelines for Use of Non-linear Analysis Methods in Documentation of Ultimate Limit States for Jacket Type of Offshore Structures, Det Norske Veritas, Norway, 1999.

[14] HKS, ABAQUS/AQUA User's Manuals v. 6.3, Hibbit, Karlsson and Sorensen Inc., Rhode Island, USA, 2002. 